\title{
Analysis of Tactical Communications Jamming Problems
}

\author{
MICHAEL J. MARCUS, MEMBER, IEEE
}

\begin{abstract}
This paper reviews the technical disciplines needed to analyze technical communications jamming problems. Such problems rarely involve pure free-space propagation so that careful attention is needed to propagation characteristics. The various sources that contribute uncertainty to the problem are enumerated and estimates are given of their magnitude.
\end{abstract}

$\mathbf{T}$ HIS paper presents a discussion of the basic factors involved in the analysis of tactical communications jamming problems. The heavy use of communications jamming in the 1973 Yom Kippur War has greatly stimulated the interest in tactical jamming within military communications and electronic warfare. Although this work was stimulated by the need to study ways of protecting communications systems from disruption, the same techniques are applicable in the study of jamming systems and in the study of protecting communications from unintentional interference.

The basic type of jamming analysis problem is shown in Fig. 1. We have a jammer and a victim pair consisting of a transmitter and receiver. In most situations, the jammer is on the opposite side of the forward edge of the battle area (FEBA) from the victims. Given all the technical details of the equipment (power, modulation, etc.) and the environment (ground conductivity, path profile, etc.), we would like to know whether the jamming is successful in disrupting communication between the transmitter and the receiver. ${ }^{1}$ As we shall see below, the proper answer to this question is often not a simple binary yes or no, but rather a probabilistic measure of success for most practical scenarios.

Many of the factors involved in jamming success are statistical in nature. In many environments, radio propagation is one of these factors. From Maxwell's equations, we can derive the fact that the electromagnetic field strength decreases as the inverse square of the distance from the source for a nonabsorptive medium, such as the troposphere at HF, VHF, and UHF. However, this deals only with free-space propagation as is usually involved in geometrical optics. For ground-to-ground, ground-to-air, and some air-to-air communications, the presence of the ground interferes with this simple model of geometrical optics due to the effect of reflected rays at the receiving antenna. This paper is not the appropriate place for a de-

Manuscript received January $15,1980$.

The author was with the Institute for Defense Analyses, Arlington, VA 22202. He is now with the Office of Science and Technology, Federal Communications Commission, Washington, DC 20554.

1 Another way to view the success of communications jamming is to ask whether it achieves a military objective. If jamming is to achieve a military objective, it must be successful in disrupting the communications link it is meant to disrupt. Thus, the disruption question forms a foundation for the broader question of military effectiveness that is being studied by others.

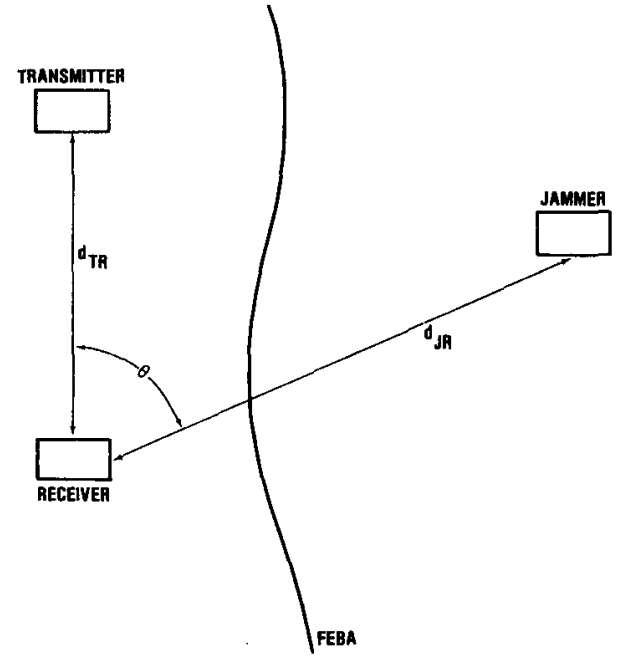

Fig. 1. Basic communications jamming analysis problem.

tailed tutorial on tropospheric radio propagation, which has been described in detail elsewhere [1], [2]. Rather, it demonstrates the use of existing models of radio propagation and other phenomena in studying communications jamming problems.

A communications jamming problem can be modeled as a series of subproblems, as shown in Fig. 2. This identifies the factors that ultimately affect the success of military operations. Ideally, we would like to be able to predict the success of military operations in terms such as exchange ratios or area of territory gained. This raises the general question of military measures of effectiveness (MOE's). A great deal has already been written on this topic within the military community. It is reasonable to state that there is no generally accepted methodology for predicting military success as a function of communication system performance. Any methodology for such a prediction must make a great many assumptions about the details of military operations and their intrinsic dependence on communications. Although some models we have seen for this have limited applications, in general, they are very sensitive to the detailed assumptions internal to the model. We shall, therefore, limit our discussion to the effectiveness of communications. This is more objective and requires many fewer assumptions in modeling.

Each block in Fig. 2 has been labeled by name and assigned a conservative estimate of the amount of variation attributable to it. First. the jammer has a variety of parameters available to it even if we know what generic type of jammer is being used. We may not know a priori what type of jamming waveform will be used (most jammers offer a choice), and we do not know how accurately the jammer will align his transmitted 


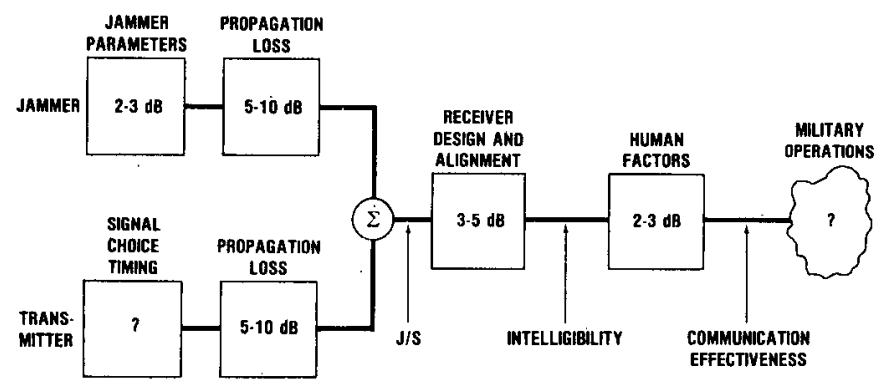

Fig. 2. Communications jamming subproblems and estimates of the amount of uncertainty each contributes.

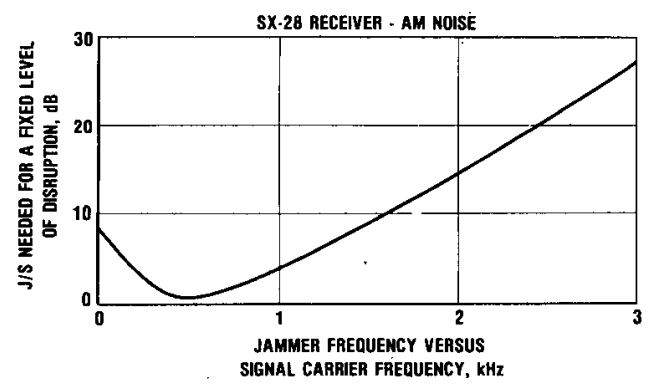

Fig. 3. Relationship between $J / S$ requirement for disruption and relative jammer and signal frequencies.

power with the frequency of the victim transmissions. In general, the effectiveness of jamming depends on the relative displacement of the center frequencies. An example of this is shown in Fig. 3, which gives the jamming-to-signal ratio $(J / S)$ needed for a certain amount of jamming effectiveness against a World War II vintage SX-28 receiver as a function of the relative carrier displacement [4] .

Propagation loss and antenna pattern variation can be the most significant sources of variation in a jamming scenario. Consider in Fig. 4 the data collected on radio path losses in measurements on the Colorado plains. These data were collècted at $101.5 \mathrm{MHz}$ in an area where the interdecile terrain height (the difference between the 90-percentile high terrain height and the 10-percentile low terrain height) $\Delta h$ was $90 \mathrm{~m}$. It can be seen that even for fixed path lengths, there were variations in path loss on the order of $20 \mathrm{~dB}$. Further studies have documented the relationship between such variations in path loss and the terrain roughness as modeled by $\Delta h$. Longley [5] has given a normal model for these path loss variations with a standard deviation $\sigma_{L}$ given by

$$
\sigma_{L}=6+0.55(\Delta h / \lambda)^{1 / 2}-0.004(\Delta h / \lambda)
$$

where $\lambda$ is the wavelength of the transmission. Table I shows appropriate values of $\Delta h$ for several common types of terrain. Values for $\sigma_{L}$ can be read from the graph in Fig. 5, where it can be seen that in the VHF and UHF bands, values from 6 to $21 \mathrm{~dB}$ are possible.

In jamming problems, one is dealing with not just one propagation path, but two: one from the transmitter to the receiver and one from the jammer to the receiver. The fact that transmission paths have a Gaussian distribution for their actual păth losses does not in itself imply that when there are two paths terminating in the same receiver, the variance of the $J / S$

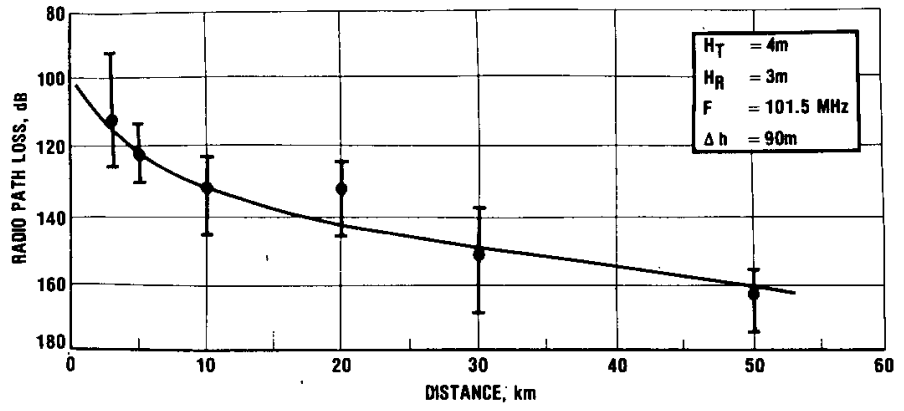

Fig. 4. Radio path loss data from Colorado plains.

TABLE I

ESTIMATES OF $\Delta h$

\begin{tabular}{lc}
\hline \hline \multicolumn{1}{c}{ Type of Terrain } & $\Delta h$, meters \\
\hline Water or very smooth plains & $0-5$ \\
Smooth plains & $5-20$ \\
Slightly rolling plains & $20-40$ \\
Rolling plains & $40-80$ \\
Hills & $80-150$ \\
Mountains & $150-300$ \\
Rugged mountains & $300-700$ \\
Extremely rugged mountains & $>700$ \\
\hline
\end{tabular}

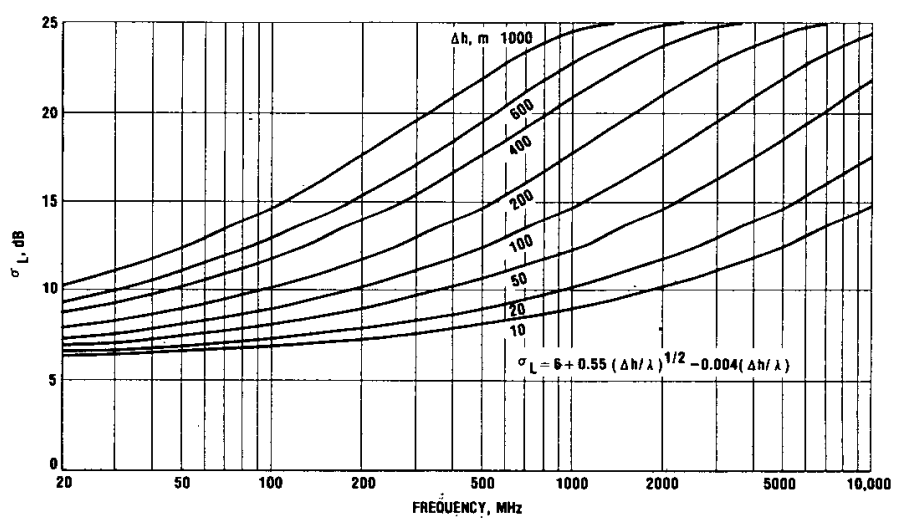

Fig. 5. Curves of $\sigma_{L}$ versus frequency for several values of terrain parameter $\Delta h$.

is the same as the variances of the two paths, as there may be correlations between the path losses. We have been unable to find a definitive answer to this question. Data presented in [6]-[8] for VHF ground-to-ground paths in Ohio and Colorado suggest that the correlation between path losses is less than 0.3 when the angle between the paths, $\theta$ in Fig. 1, is greater than $45^{\circ}$. For $0^{\circ}<\theta<45^{\circ}$, correlation values varying from 0.1 to 0.7 have been found. On the basis of these data and discussions with personnel of the Institute for Telecommunications Sciences, we feel justified in modeling the two path losses as being independent. If more definitive data should become available, however, it would be useful to model the small effect of path loss correlation, especially for situations in which $\theta$ is small.

For some classes of problems, the antennas used have very 


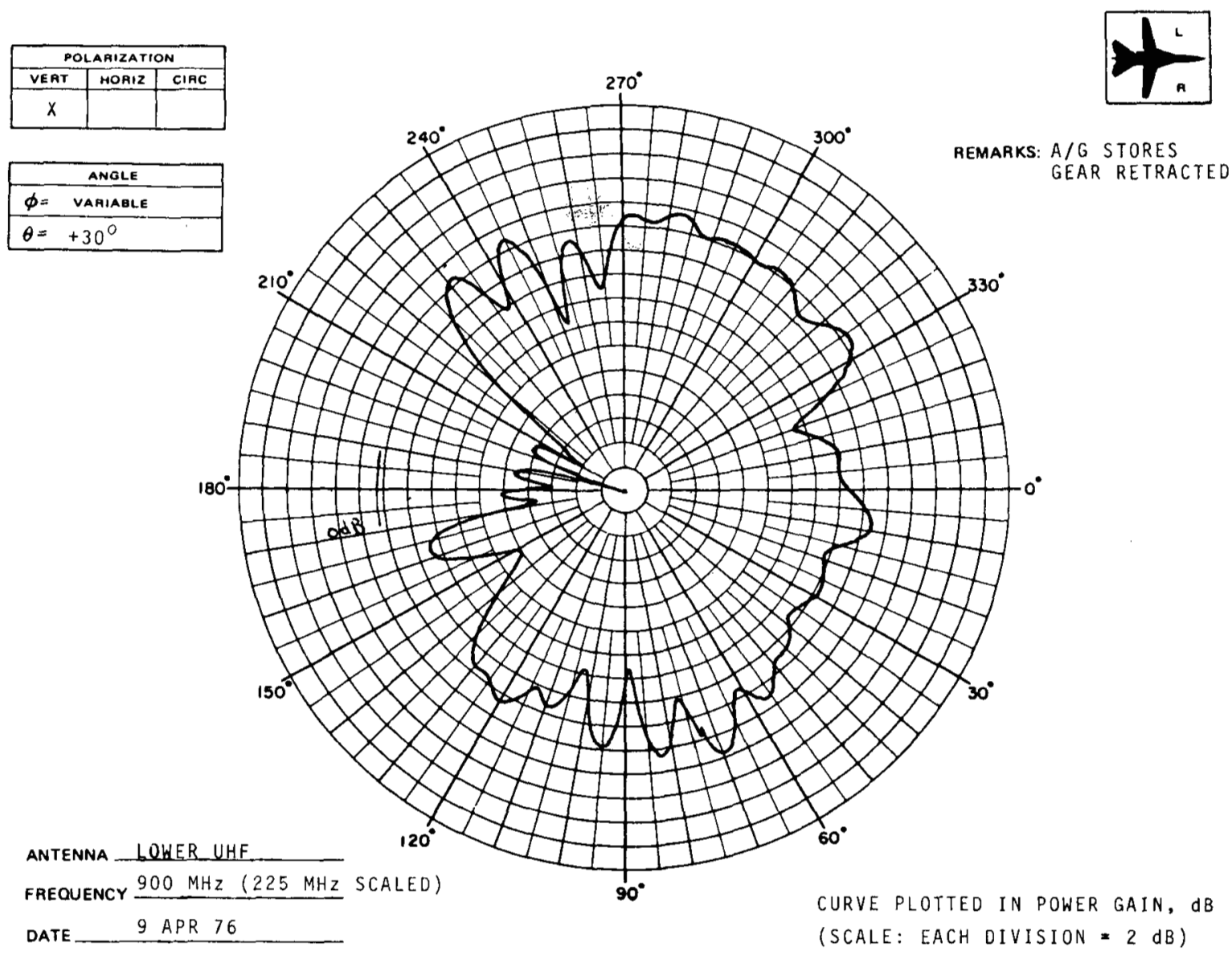

Fig. 6. Antenna pattern of a typical tactical fighter with externally mounted munitions and landing gear retracted.

complicated radiation patterns that contribute to the overall uncertainty in the analysis. For example, in problems involving aircraft, the detailed microstructure of the radiation pattern from the aircraft's antenna is impractical to model deterministically and is best modeled as a stochastic process. The antenna patterns are a function of antenna type, landing gear status, location on aircraft, aircraft frame design, amount and location of external stores (missiles, bombs, tanks, etc.), and frequency. Typical antenna patterns are usually measured during the aircraft's development cycle by using scale models at scaled frequencies. One such pattern for a typical tactical fighter is shown in Fig. 6. It can be seen that there are several decibels variation in the radiation pattern as the azimuth changes. Since the radiation pattern is a function of so many variables (and can change during a mission due to release of stores), it is not possible to model it deterministically; rather, a statistical model is more appropriate. In modeling the variation in antenna patterns, we have used a normal distribution whose standard deviation is approximately the roughness of the antenna pattern, $4 \mathrm{~dB}$ in the case of Fig. 6.

At the antenna of the victim receiver, the desired signal and the jamming signal are combined, and it is conceptually possible to measure the $J / S$. If all measurements are done in decibels and variations are modeled as normal variables, their variances will add at this point, so that the standard deviation here will be 7-14 dB.
Jamming will be effective if there is enough jamming power to exceed the jamming threshold of the receiver and its human listener. We consider the receiver and the human separately, as different factors affect them. The net effect of these factors is that the jamming threshold is also a random quantity that must be modeled statistically. If the jamming threshold is modeled as a normal distribution, the formulation is straightforward for

$$
\operatorname{Pr}[J / S>\text { threshold }]=\operatorname{Pr}[J / S-\text { threshold }>0] .
$$

We can define a new random variable ( $J / S$ - threshold) whose mean is the difference of the means of $J / S$ and threshold and whose variance is the sum of their variances. For the normal model this can be evaluated using error functions.

The ability of a given $J / S$ to degrade a receiver depends upon both the basic design of the receiver and its current state of maintenance (in particular, the front-end alignment). These affect the intermediate frequency (IF) bandwidth, which determines how much of the jamming-and-signal combination passes to the receiver's detection circuit. This relationship is demonstrated in Fig. 7, which shows the $J / S$ (measured not in terms of total jammer power, but jammer power density/hertz) needed to degrade receiver performance to a given point as a function of IF bandwidth. When studying offensive jamming problems, the analyst does not always know 


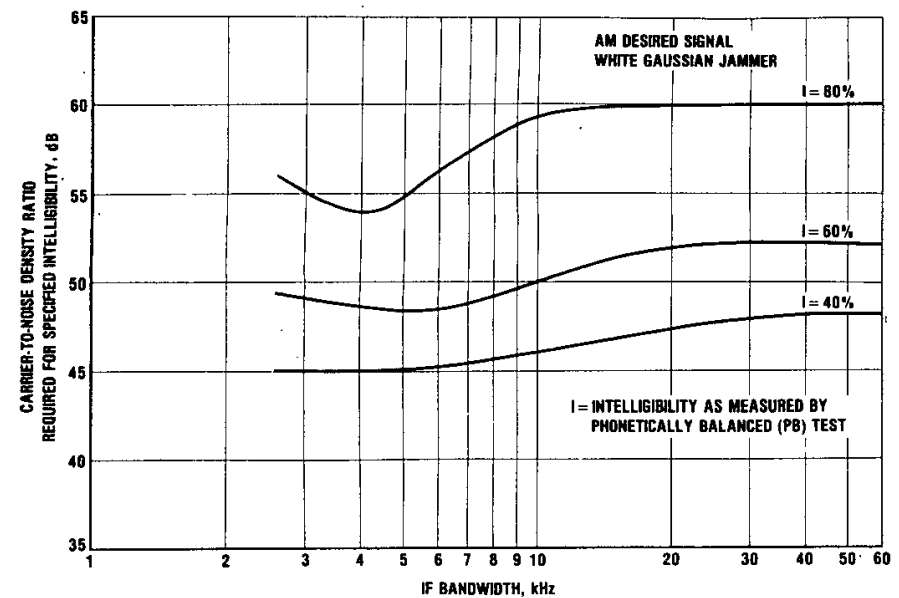

Fig. 7. Effect of IF bandwidth on jamming. (Data source: [9].)

exactly what receiver the victim may be using or what its detailed technical characteristics are. However, in both offense and defense the analyst must be aware that receivers are seldom maintained so as to meet manufacturers' specifications. Two identically nomenclatured receivers may have different bandwidths due to differences in alignment. Therefore, the uncertainties in the victim receiver's front end can add a few decibels of uncertainty to the jamming threshold.

Just as the input to a receiver can be characterized in terms of $J / S$, the audio output can be characterized in terms of a psychoacoustic measure called intelligibility. However, where $J / S$ can conceptually be measured with a meter, intelligibility is a more elusive quantity. MIL-STD-1472B [10] recognizes two different techniques for determining intelligibility. A great many others have been described in the literature. These measures do not describe the probability that a message was received correctly, but the probability that a single consonant or word was discerned correctly when a trained listener heard a series of words in a prescribed format under laboratory conditions. Thus, intelligibility is not readily measured under field conditions nor is it precisely reproducible. Nevertheless, when correctly interpreted, it is a useful tool in studying jamming.

Given the intelligibility of the signal presented to the listener, we still cannot completely judge whether communications will be successful. The experience, motivation, and composure of the listener are very important factors in determining the success of communications. Unfortunately, quantitative data in this area are scarce, although combat experience has shown the worth of trained, experienced operators [4]. However, Fig. 8 shows data from one experiment in this area involving jamming of $\mathrm{AM}$ transmissions which caused speech inversions. These data indicate that for AM systems, the experience of the operator can affect the jamming threshold by more than $10 \mathrm{~dB}$ in some cases. Thus, analysis must allow for some uncertainty as to the victim's training and condition.

It is interesting to note here that it is often thought that FM systems have a binary condition of being either jammed or not jammed due to the "FM capture effect," while AM systems degrade more gracefully with increased jamming. Figs. 9

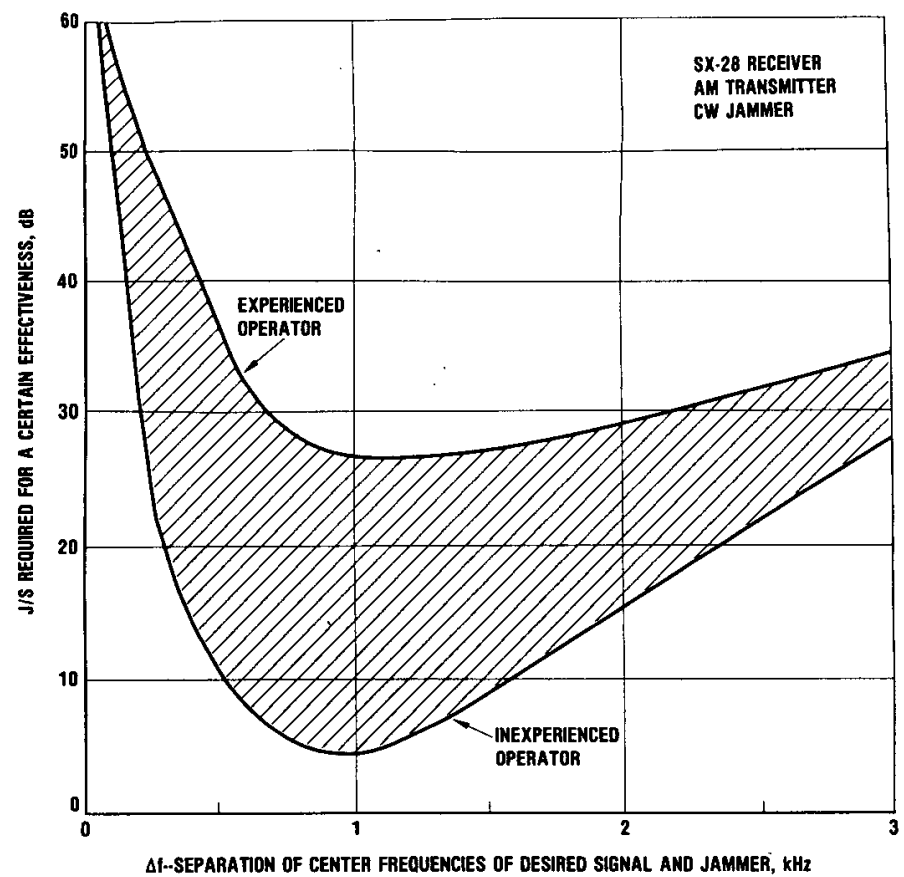

Fig. 8. Effect of operator experience on jamming effectiveness.

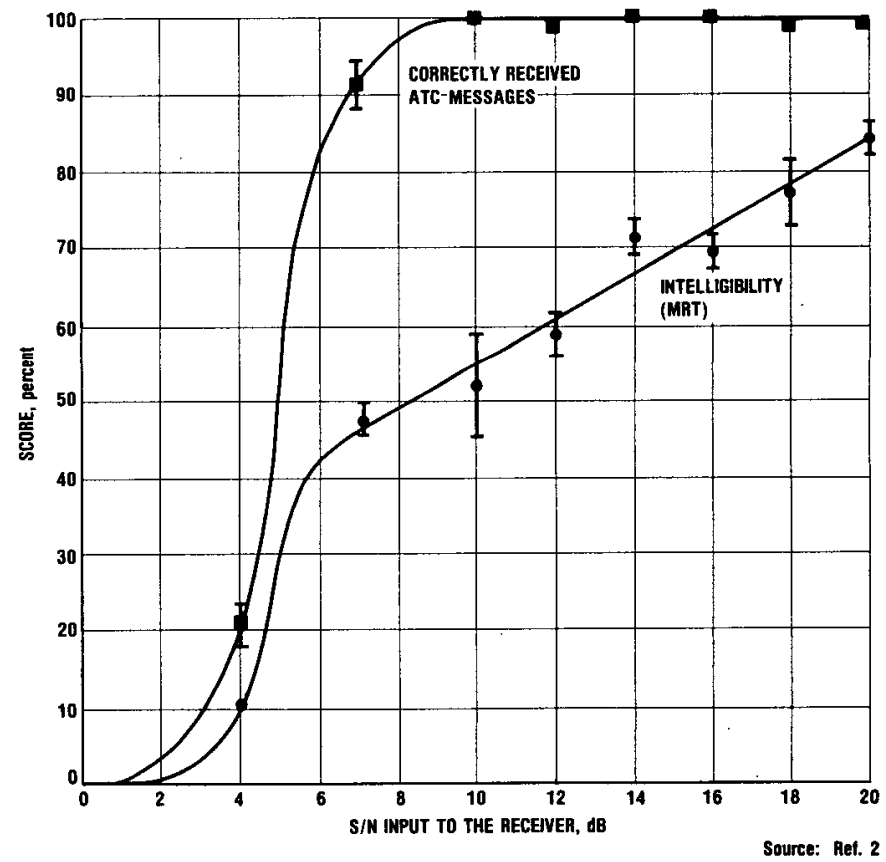

Fig. 9. FM performance versus wideband noise. (Source: [2].)

and 10 show data collected by the Institute for Telecommunications Sciences on this question. It can be seen that, as expected, FM intelligibility has a steeper slope with $S / N$ than does AM intelligibility. However, when performance is quantified as to the fraction of actual messages correctly received-in this case, air traffic control (ATC) messages-both AM and FM have very steep slopes, and for a given set of circumstances, a system either works or does not. Thus, it seems reasonable to 


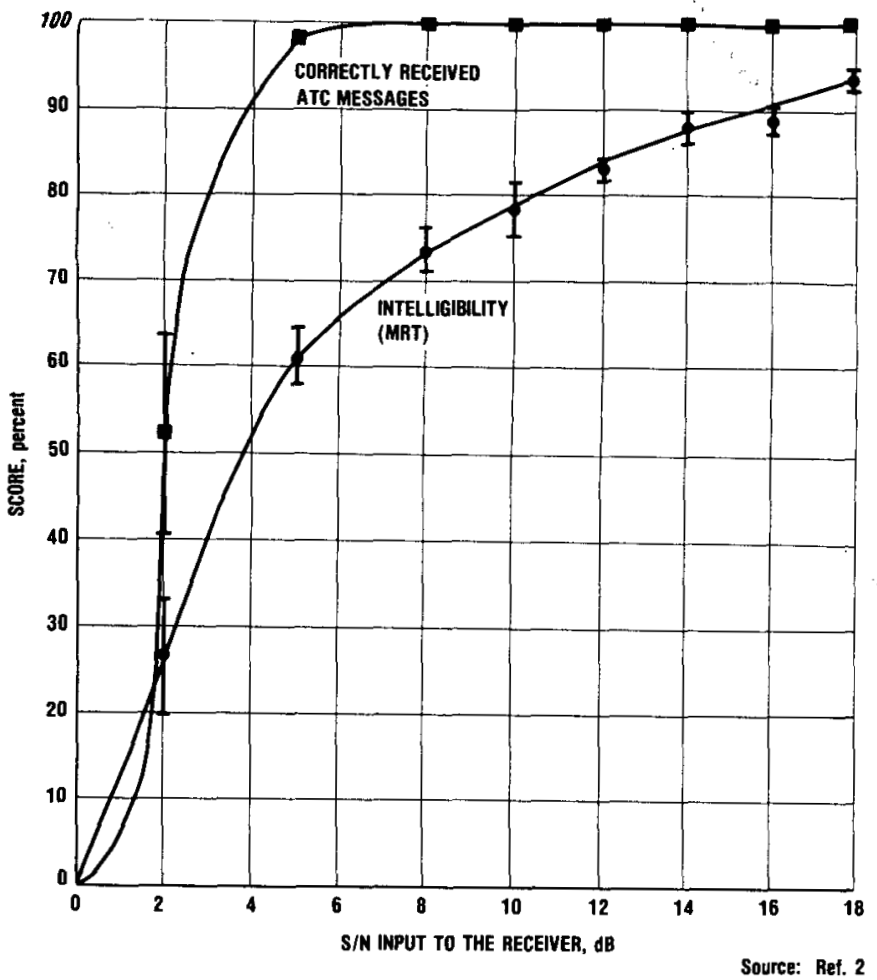

Fig. 10. AM performance versus wideband noise. (Source: [2].)

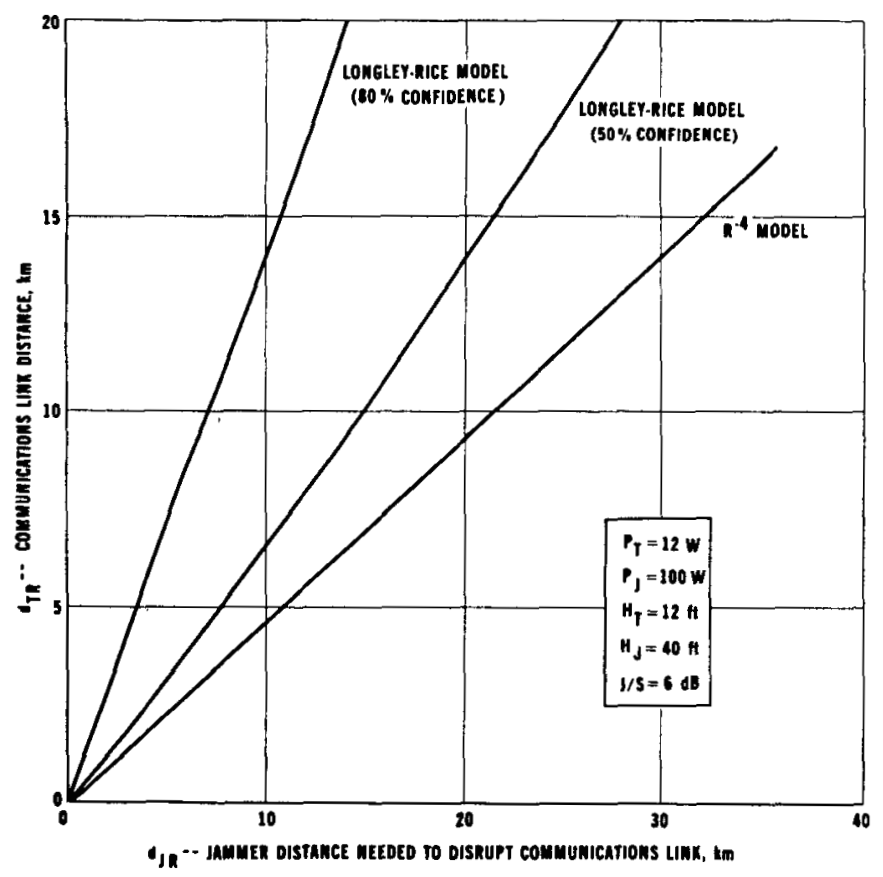

Fig. 11. Comparison of propagation models.

model both $\mathrm{AM}$ and FM systems as having thresholds for $J / S$ at the receiver input, above which communication is not practical. Both the receiver $J / S$ level and the threshold must be modeled statistically for the various reasons given above. If estimates are made of the variances of each of the subproblems shown in Fig. 2, these can be added to compute a total variance of the overall problem, $\sigma_{T}$. Jamming will occur if the $J / S$ exceeds the threshold of the system. Assuming normal distributions for all the variations, where we obtain

$$
\operatorname{Pr}\{\text { jamming }\}=\operatorname{Pr}\left\{N\left[\left(\frac{\bar{J}}{\bar{S}}\right)-\text { threshold, } \sigma_{T}\right]>0\right\}
$$

where $N[\mu, \sigma]$ is a normal variate with mean $=\mu$, variance $=$ $\sigma^{2}$. This can be evaluated readily for actual problems using either tabulated error functions or error function subroutines.

Using these concepts we shall give an example of jamming analysis. Fig. 11 shows the relationship between $d_{T R}$ and $d_{J R}$ for a situation where the jammer is $100 \mathrm{~W}$ and the transmitter is $12 \mathrm{~W}$. It is assumed that a $J / S$ ratio of $6 \mathrm{~dB}$ will disrupt communications, although the exact requirement will be a function of radio and jammer design. The area to the left of the line labeled $R^{-4}$ model is where jamming would be effective if it was assumed that field strengths were deterministic and were a function of the inverse fourth power of range (an approximate model for propagation over a flat earth). The other two curves show the results obtained using the LongleyRice propagation model. For these curves it was assumed that the carrier frequency was $40 \mathrm{MHz}$ and the terrain roughness, $\Delta h$, was $100 \mathrm{~m}$, yielding a location variability, $\sigma_{L}$, from Fig. 5 , of $8 \mathrm{~dB}$. Assuming that the location variabilities of the transmitter and jammer path are independent, the total $J / S$ variability $\sigma_{T}$ is then $11.3 \mathrm{~dB}$. The two Longley-Rice curves show the relationship between $d_{J R}$ and $d_{T R}$ for 50 and 80 percent confidence of $J / S>6 \mathrm{~dB}$. Thus, if a military commander wants an 80 percent chance of disrupting an enemy circuit, he must be prepared to have his jammers much closer than if he can accept a 50 percent chance. The use of the $R^{-4}$ model can be seen to give very misleading results.

\section{CONCLUSIONS}

The analysis of communications jamming problems involves many different aspects of communications engineering, such as radio propagation, antenna characteristics, receiver design, and intelligibility testing. For tactical VHF and UHF scenarios, the effectiveness of jamming can only be predicted statistically, not deterministically, due to statistical variations in the factors shown in Fig. 2. These modeling concepts should also be useful in the analysis of unintentional interference problems.

\section{REFERENCES}

[1] P. L. Rice et al., "'Transmission loss prediction for tropospheric communication circuits," Inst. Telecommun. Sciences Aeronomy, Nat. Bureau Standards Tech. Note 101, AD 687820 and AD 687 $821,1967$.

[2] A. G. Longley, "Electrospace planning and engineering for the air traffic environment," Inst. Telecommun. Sciences, Rep. FAA-RD70-71, AD 718 447, Dec. 1970

[3] RCM Handbook No. 4-Communications Countermeasures, Nat. Defense Res. Committee, AD $130274,1945$.

[4] "Location variability of transmission loss--Land mobile and broadcast systems," Inst. Telecommun. Sciences, OT Rep. 76-87, May 1976. 
[5] M. J. Miles, "The effect of irregular terrain and atmosphere on correlation between transmission loss values," Inst. Telecommun. Sciences, ESSA Tech. Rep. ERL84-ITS69, June 1969

[6] R. E. Skerjanec, "A note on measured spatial correlation coefficients between VHF signals received in irregular terrain," Inst. Telecommun. Sciences, Tech. Memo. ERLTM-ITS197, Aug. 1969.

[7] A. P. Barris and M. J. Miles, "Height gain, location gain, and correlation in irregular terrain at 20,50 , and $100 \mathrm{MHz}, "$ Inst. Telecommun. Sciences, IER35-ITSA35, AD 655 901, Apr. 1967.

[8] J. Cunningham, S. J. Goffard, and J. C. R. Licklider, "The influence of amplitude limiting and frequency selectivity upon the performance of radio receivers in noise," Proc. IRE, vol. 47, pp. 1021-1025, Oct. 1947.

[9] "Military standard-Human engineering design criteria for military systems, equipment, and facilities," Dep. Defense, MILSTD1472B, Dec. 31, 1974.

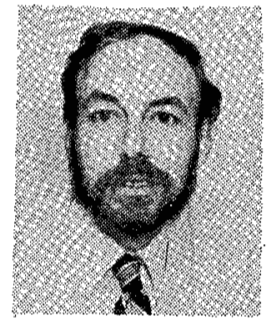

Michael J. Marcus (S'66-M'72) was born in Boston, MA, on May 13, 1946. He received the S.B. and Sc.D. degrees in electrical engineering from the Massachusetts Institute of Technology, Cambridge, in 1968 and 1972 , respectively.

While an undergraduate at M.I.T., he participated in a cooperative program at Bell Telephone Laboratories and continued as a consultant until 1971 in the area of telephone switching theory. From 1972 to 1975 he served in the U.S. Air Force as a project officer for underground nuclear test detection research. He then joined the Institute for Defense Analyses, where he was involved in communications ECCM requirements analysis and computer communications network design. In September 1979 he joined the Federal Communications Commission as Special Assistant to the Chief Scientist for Technology Assessment.

Dr. Marcus is a member of Eta Kappa Nu, Tau Beta Pi, and Sigma Xi.

\title{
IFF System Concept Based on Time Synchronization
}

\author{
WILLIAM M. BRIDGE, MEMBER, IEEE
}

\begin{abstract}
Identification of friend or foe (IFF) in the tactical theater is a complex technical and operational problem with extremely important implications to the nation's success in waging war. A practical, inexpensive IFF system which can reliably identify friends in a hostile ECM environment is desperately needed by the United States and NATO. This paper presents an IFF system concept which utilizes existing technologies and devices to create a new tactical IFF system that addresses the following electronic counter-countermeasure (ECCM) problems:

1) vulnerability to enemy antiradiation missiles,

2) vulnerability to jamming,

3) vulnerability to enemy spoofing and deception, and

4) vulnerability to enemy exploitation ("eavesdropping").

The system concept is based on accurate time-synchronization among all participants and represents a radical departure from current IFF methods. However, it does retain the basic interrogateresponse approach. It utilizes spread-spectrum, low-data-rate signaling which offers substantial resistance to enemy jamming and makes spoofing and deception extremely difficult. The spreadspectrum techniques include: frequency hopping, time jitter, and intrapulse coding. This combination of techniques is used to maximize the antijam and convert capabilities of the system without putting undue burden on a single technology.
\end{abstract}

\section{INTRODUCTION}

\section{Background}

DENTIFICATION of friend or foe (IFF) in the tactical 1 theater is a complex technical and operational problem with extremely important implications to the nation's success in waging war. A fundamental military need is that friends, allies, and neutrals must be identified so that they will not be attacked

Manuscript received January 15, 1980. This paper was presented at the Naval Research Laboratories Identification of Friend or Foe Symposium, June 12, 1980.

The author is with the MITRE Corporation, Bedford, MA 01730. by friendly weapons. A second fundamental need is the identification of enemies, so that we may commit our weapons against them in a timely and effective manner.

Although the basic IFF problem has been an element of all wars, the situation is rapidly becoming aggravated by various technological advances. Guided missiles small enough to be carried by a single infantryman are now capable of destroying armored vehicles and high-performance aircraft. New air-toair and ground-to-air missiles have been developed with lethal ranges greater than $50 \mathrm{mi}$. Weapons, sensor systems, and tactics have been combined in low-altitude "pop up" maneuvers which give little time for recognition and decision. Adversaries have grown increasingly sophisticated in exploitation of our IFF systems (to identify and locate our aircraft), in electronic spoofing and jamming, and in the development of antiradiation missiles for the destruction of IFF transmitters.

The current IFF system has many failings which have been recognized and debated within the IFF community for years. The system is actually not capable of true IFF. At best it can only detect the presense of properly equipped friends but cannot positively identify enemies or unequipped neutrals. Its overall reliability is poor, especially in a hostile ECM (electronic countermeasure) environment and it is operationally awkward in its cryptographic mode.

\section{Purpose}

A practical, inexpensive IFF system which can reliably identify friends in a hostile ECM environment is desperately needed by the United States and NATO. This paper presents an IFF system concept which utilizes existing technologies and devices to create a new tactical IFF system that addresses the following electronic counter-countermeasure (ECCM) 